\title{
Simulation of a Panel Positioning Robot
}

\author{
Javier Ibañez-Guzmán, Brian Atkin and Peter Atkinson \\ Construction Robotics Research Group, University of Reading, \\ Whiteknights, PO Box 219, Reading, RG6 2BU, United Kingdom
}

\begin{abstract}
This paper describes the work and results of a feasibility study into the simulation of a panel positioning robot. A kinematic model was built, using GRASP and ROBCAD software, to simulate robot motion and to determine optimal configuration, cycle times and work envelopes. A dynamic model was then built, using TUTSIM, in order to determine the robot's dynamic response to payloads and disturbances. Test-runs show that the configuration of the robot and its control strategies/servo-mechanisms are feasible, allowing the robot to work alongside a human operator in positioning and fixing panels onto a building's structure. Data generated by the test-runs have helped to advance the design of the device and are presented.
\end{abstract}

\section{INTRODUCTION}

The design and implementation of a construction robot is potentially expensive (both in time and resources), requiring a team of specialists and facilities for performing operational tests on the device. The use of computer assisted design and simulation techniques, however, enables different robot configurations and control schemes to be evaluated without extensive set up times and costs.

This paper describes the simulation techniques and the models developed as part of a feasibility study carried out on a panel (cladding) positioning robot. It includes specifications and modifications to the mechanical design obtained from test-runs performed on the robot model. For the purposes of this study, kinematic and dynamic models were developed. Computer assisted control system design techniques were used for designing the servo controllers of the robot links.

The authors have already argued that construction projects should be seen as an integrated process, consisting of a set of complementary subsystems none of which can be automated in isolation ${ }^{1,2}$. Moreover, the physical operations which would be performed by robotic/automated equipment are the end-product of a series of processes. Moves towards automating construction must therefore consider all processes, not just the physical operations at which a potential robotic device would be aimed but also the building components which would need to be manipulated ${ }^{3}$. In this connection, the authors have proposed a family of robotic devices to assist in modified, on-site construction processes $\mathrm{s}^{4,5}$. Of the devices proposed, a cladding positioning robot was selected for the feasibility study.

The following sections describe the panel positioning system, the simulation techniques employed and the kinematic and dynamic models produced, together with their control strategies. Thus, the objective of this paper is to report the results of the feasibility study.

\section{PANEL POSITIONING SYSTEM}

Today, buildings are clad in a variety of materials including glass, aluminium and steel alloys. Cladding panels are often man-handled up ladders and along platforms by operatives and then held in position whilst fixed. The positioning and fixing of panels could be automated by means of the panel positioning system shown in Figure 1. This comprises an external access work platform, a panel positioning robot and a rough-terrain, fork-lift truck. 


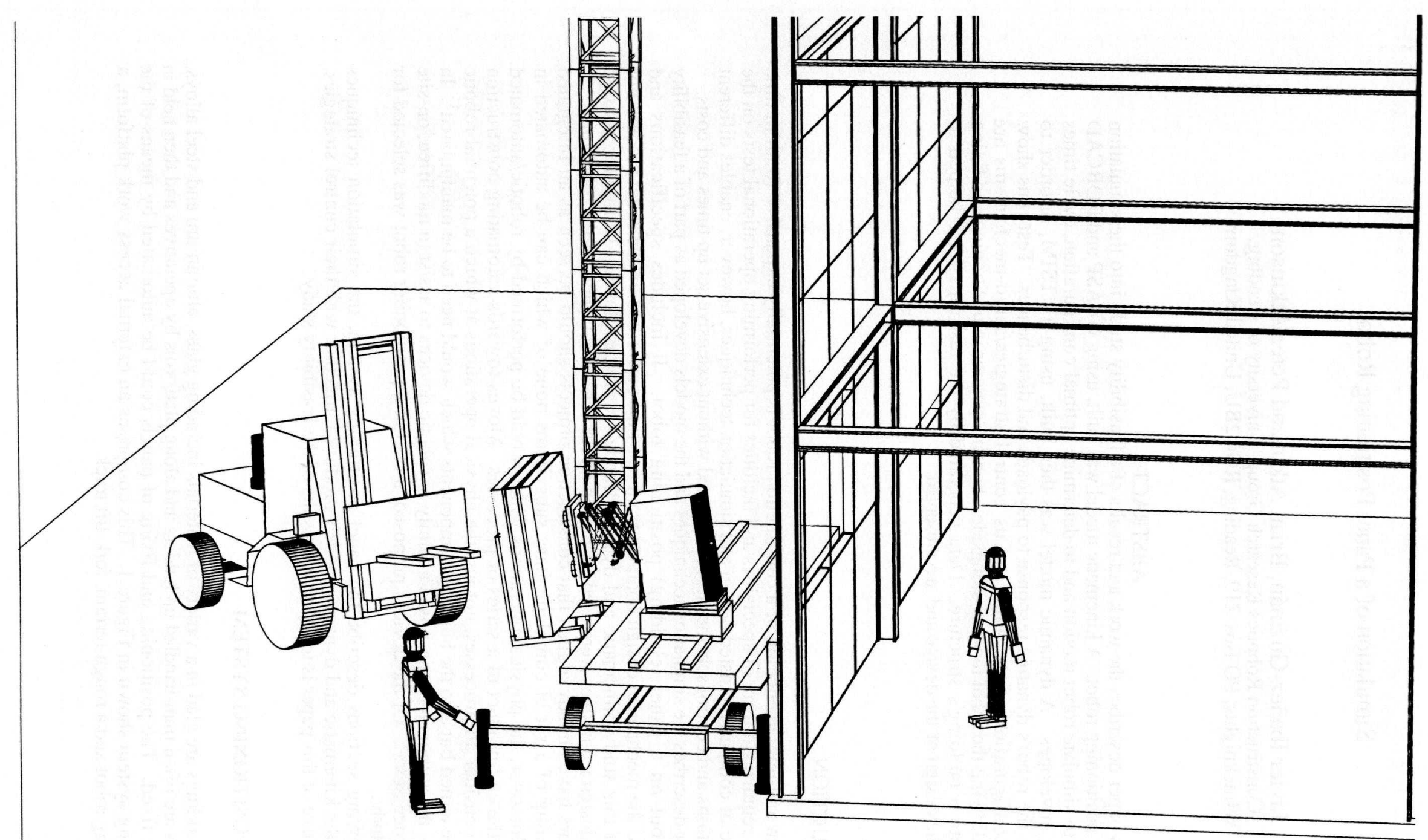

Figure 1. Panel Positioning System 
Consignments of panels are delivered to the stock-cradle, located on one side of the work deck of the platform, using a rough-terrain, fork-lift truck. Once loading of the panles has been completed, the cradle is then raised to the required level under operator control. The operator positions the robot in front of the panels held in the cradle. The manipulator arm then moves forward under robot control until the suction cups are in close contact with the panels. On contact, the suction cups are activated to pick up a panel. The robot controller commands the manipulator arm to move backwards and to rotate the robot body: the swivel motion moves the panel from its previously angled position to a vertical position parallel to the cladding rails on the building. The manipulator arm of the robot now moves the panel automatically towards its approximate final position. When this is reached, the operator takes control using a pair of joysticks. Thus, final positioning of the panel is made under operator control from a location within the building close to the panel. Once the desired position for the panel has been reached, the robot holds it in place whilst the operator achieves a temporary fixing. A signal to release the panel is then sent to the robot by the operator. The robot then proceeds to pick and place the next panel. The platform can either be raised to a new level or moved along the building to its next reference position.

Details of the maximum/minimum panel sizes and payloads manipulated by the robot are included in section 4 as part of the results obtained from the simulation test-runs. The type of actuators, their sizing and control strategies are described in section 5.

\section{RATIONALE FOR SIMULATION}

Simulation is a well-defined engineering tool for design analysis and the control of complex, physical systems. There are many situations in which a computer simulation, coupled with a graphical display, can be very useful in the design of a robotic system 6,7 . Simulation tools can also be used to design an entire robotic system (that is robot and peripherals) and to study ways of integrating the robot with the project of which it forms a part. The graphical aspect of the simulation can be used to represent the workspace of a given geometric configuration without the need for building a physical, scale model. The control engineer can also use the data generated from simulations to size motors and develop control system strategies for the robot. Of course, graphical output will also be used by the control engineer, since graphical displays can convey more information to the designer than alphanumeric data alone ${ }^{8}$.

The design and feasibility study of the proposed panel positioning system would ordinarily be an expensive exercise, requiring several specialists and facilities for carrying out tests on the prototype. The size of the system - the length of the work platform alone is $6.0 \mathrm{~m}$ - and the scale of the working environment makes prototypying very difficult, especially in a university. Thus, simulation techniques enable different robot configurations and control schemes to be evaluated timeand cost-effectively.

Two simulation models were built. The first, a kinematic model, was used as a test-rig on which the robot's configuration and specification were determined. The second, a dynamic model, was used to simulate the response of the robot to variations in payloads, but unlike simplified laboratory testing was able to include disturbances such as wind gusting. The dynamic behaviour of the robot was observed under automated and teleoperated operation.

\section{KINEMATIC MODEL}

A kinematic model of the robot was built in order to simulate robot motion and to determine optimal configuration, cycle times and work envelopes. It was also used to check for potential collisions. Kinematic simulation shows the motion of the robot though without considering how it is achieved by actuator torques and forces ${ }^{9}$. To this end, 3-D graphics simulators were used, GRASP 10 and ROBCAD ${ }^{11}$, to create a computer animation of the panel positioning/fixing process.

The model of the robot was obtained by translating its structure into the language of the simulator. A unique description and coordinate system was assigned for each degree of freedom of the robot, with the relationship between the neighbouring coordinate systems being defined in terms of their position and orientation. The type of degree of freedom (revolute or prismatic) had also to be 
described, together with its direction of motion. Finally, the distance/angle which every joint is allowed to move and maximum/minimum velocities and accelerations were included. It should be emphasised, however, that these parameters specify the kinematic structure of the robot only.

\begin{tabular}{|l|l|l|l|}
\hline Joint & Position & $\begin{array}{l}\text { Type- } \\
\text { Direction }\end{array}$ & Observations \\
\hline 1. Work deck & $\mathrm{z} 6250$ & $-\mathrm{Z}_{1}$ & Electric \\
2. Robot base & $\mathrm{y}-1150, \mathrm{z} 220$ & $-\mathrm{X} 2$ & Electric \\
3. Swivel & $\mathrm{z} 170, \varnothing \mathrm{x}-4$ & $-\varnothing_{3}$ & Electric yaw \\
4. Arm & $\mathrm{y} 890, \mathrm{z} 620, \varnothing \mathrm{x}=-4$ & $\mathrm{y} 4$ & Hydraulic \\
5. End-effector & & $\emptyset_{\mathrm{x}-5}$ & $\begin{array}{l}\text { Compliance } \\
\text { arm/cups pitch }\end{array}$ \\
6. Work object & & $\varnothing \mathrm{y}-6$ & Panel fixings roll \\
\hline$\varnothing==$ revolute & $\mathrm{x}, \mathrm{y}, \mathrm{z}==$ prismatic
\end{tabular}

Table 1. Kinematic Description of the Robot

Table 1 shows the principal characteristics of the robot's kinematic structure, indicating the type of actuator used for each joint. The robot is a four degree of freedom manipulator, that is a sort of cartesian robot with an additional yaw motion. The extra degrees of freedom required to orientate the panel in space are provided by the compliance of the lazy tongs and suction cups (pitch) and the specially-designed fixings for the cladding panels ${ }^{3}$ (roll). In addition, it was considered necessary to include a kinematic description of the two remote (external) axes which drive the stock-cradles and the platform safety guard.

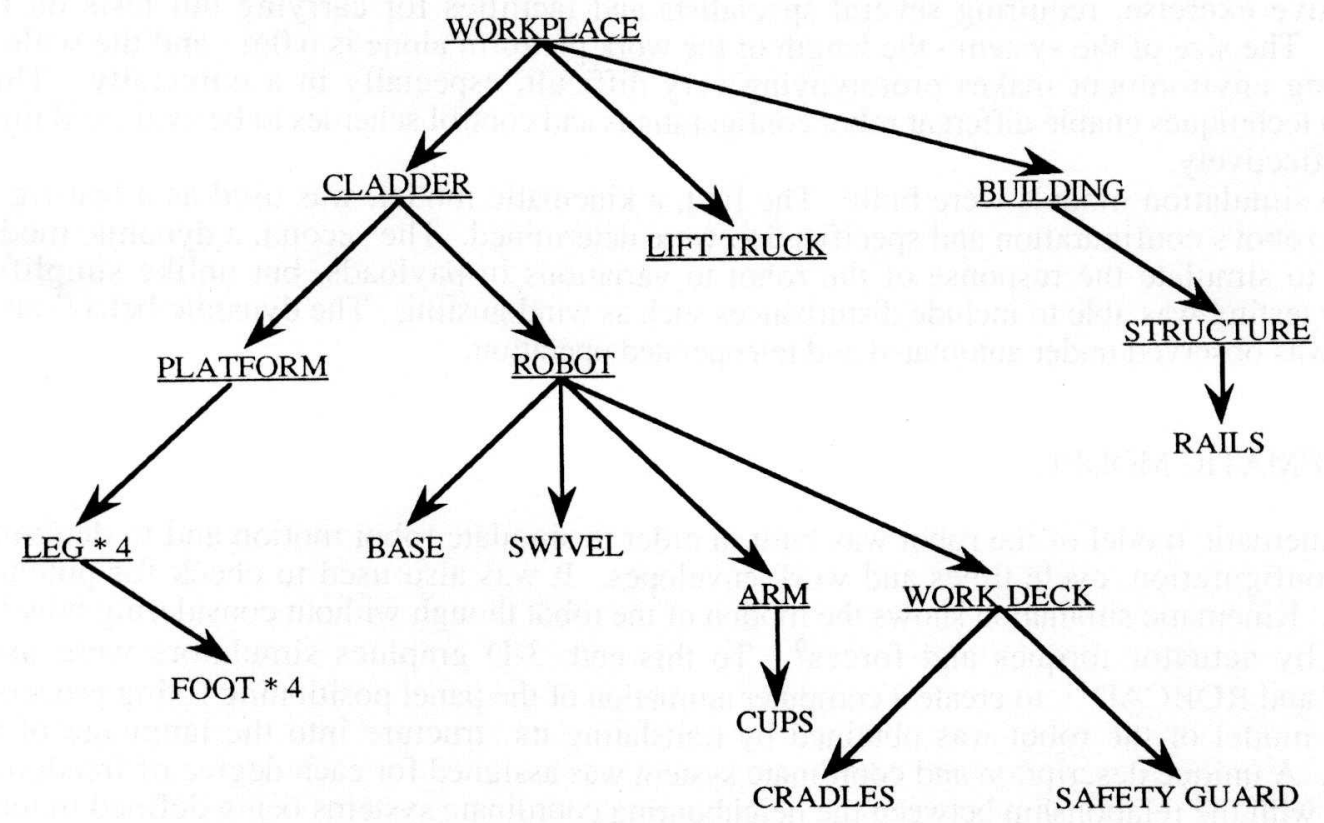

Figure 2. Model of the Test-Runs 
In order to complete the kinematic model, a description of the physical structure of the robot was added. The volumes occupied by the different links, actuators and other system components were thus designed and incorporated into the model. These details were needed to enable collision detection to be performed and for generating a realistic graphical display.

The robot was integrated into its working environment through the use of the software referred to above. The model included the peripheral equipment necessary for the operation of the robot, namely the rough-terrain, fork-lift truck, and the building structure with cladding rails. A tree of the world model for the test-runs is shown in Figure 2.

Test-runs of the robot enabled the final position of every joint to be determined: these data were shown in Table 1. The initial results gave the range of panel sizes which the robot can manipulate: these are shown below in Table 2 .

\begin{tabular}{|l|l|l|}
\hline Panel & $\begin{array}{l}\text { Allowed Sizes } \\
(\mathrm{mm})\end{array}$ & $\begin{array}{l}\text { Tolerances } \\
\text { (worst case) }\end{array}$ \\
\hline Width (x) & $700 \leq \mathrm{x} \leq 1900$ & roll $\partial \mathrm{r}=1^{\circ}$ \\
Depth (y) & $40 \leq \mathrm{y} \leq 180$ & pitch $\partial \mathrm{p}=1^{\circ}$ \\
Height (z) & $700 \leq \mathrm{z} \leq 2000$ & yaw $\partial \mathrm{y}=1^{\circ}$ \\
\hline
\end{tabular}

Table 2. Allowable Panel Sizes and Permitted Tolerances

Simulations were made of the entire operational cycle of the system using various combinations of panel sizes. Several modifications were necessary to the mechanical design in order to accommodate as wide a range of panel sizes as possible. Collision detection was of particular concern at this stage.

A study of positioning tolerances was made in order to determine the maximum misalignment errors between the position/orientation of the panel given by the robot and the cladding rails on the building. Results of the worst case combination of panel size and building misalignments are shown in Table 2. For the purpose of this study, the effects of structural bending of the work platform and robot arm were determined and taken into account.

In order to determine the cycle times of the robot, the maximum/minimum velocities and accelerations of every joint had to be determined. The calculation of these parameters was dependent upon the maximum suction force attainable by the suction cups on the end-effector, the maximum panel load and the structural characteristics of the robot. Thus, it was necessary to determine these values first: the maximum allowed payload per panel is $90 \mathrm{~kg}$ with a safety factor of 2 .

Cycle times are dependent upon panel size and the height of the wall which the robot is intended to cover. With the correct robot configuration, a sample time taken to position and fix panels into an area of $15.60 \mathrm{~m}^{2}$ (using cladding panels of $\mathrm{W}=1.5 \mathrm{~m}, \mathrm{H}=1.70 \mathrm{~m}, \mathrm{~T}=0.15 \mathrm{~m}$ ) was 52 minutes, including set up times for the work platform and loading the panels into the stock-cradles. A fixing time of 2 minutes per panel was used in the calculations. This result means that the system would cover a wall at an approximate rate of $0.3 \mathrm{~m}^{2} /$ minute.

\section{DYNAMIC MODEL}

The kinematic model, whilst providing much useful data for the detailed design of the robot, does not take account of dynamic responses arising from payloads and disturbances. It was thus necessary to build a dynamic model of the robot.

The use of coordinated motion for all axes, enabling the robot to reach the position commanded, implies that the dynamic equations describing its motions would be non-linear and highly coupled. The close-form analytical solution of these equations is not however available ${ }^{12}$. The coupling terms represent gravitational torques, which are dependent on joint positions, and coriolis and centrifugal forces. The combination of these effects indicates that the control system for 
coordinated motion would needed to be very advanced. One of the objectives in the design of this robot was to produce a simple machine. Consequently, the mechanical design and operational sequence was developed for single-axis motion only. Treating each joint of the robot as a simple servo-mechanism was intended to simplify robot-control design ${ }^{9}$.

The proposed robot control system configuration consists of a global control system, a man-machine interface, communication links with the platform control system and joint servo-mechanisms, and system perception devices. Figure 3 shows the configuration of the robot main control system with a list of its functions.

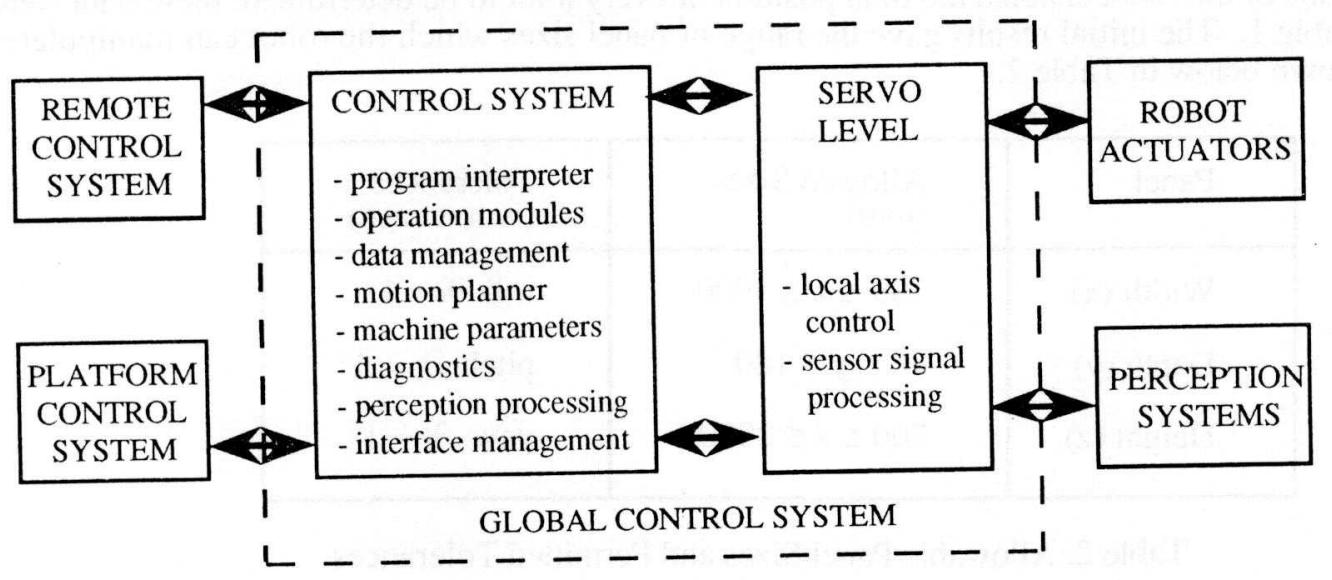

Figure 3. Robot Main Control System Configuration

By determining the most appropriate control strategies and the type of robot motion (single-axis), it was possible to build the dynamic model. The size of the actuators, gearbox (where applicable) and the type of sensors required for every joint were determined. The next stage consisted of a preliminary structural analysis giving the physical characteristics of the various mechanical components. These data were used in calculating the masses moved by the joints.

The main disturbance acting on the robot is the effect of wind gusts. Designs and simulations were produced using a recommended ${ }^{13}$ wind load factor of $1 \mathrm{KN} / \mathrm{m}^{2}$. Wind loads were modelled as a bell-shaped function to which random noise was added. Worst case conditions were considered using the largest panel sizes.

The design of the servo-mechanisms was achieved by treating each joint separately. The basic control principle is simple: move the system in the direction that minimises error ${ }^{14}$. The initial approach considered the difference between the required and actual position of the joint as an error function. It was then possible to apply a signal that was proportional to the error needed to achieve control (Proportional Control) ${ }^{15}$. However, holding a payload against the effects of wind causes a steady-state error. An alternative approach is to make the error signal proportional to the integral of the error (PI) with respect to time. In this case, the integration term of the controller reduces the steady-state error, but tends to increase overshoot when joint motion reaches high speed. So as to decrease the overshoot effect of the integration term, the drive torque is made proportional to the derivative of position (PD) with respect to time. The derivative feedback is combined to arrive at PID control. This is the type of control scheme used for each joint of the robot, except for the hydraulic actuator used to move the lazy tongs. Here, only PD control is used. This exception is due to the speed of the hydraulic actuator which is difficult to measure because of the small distances covered; taking the derivative of the position would not be particularly effective.

Simulation of each servo was undertaken using the TUTSIM dynamic simulation program ${ }^{16}$. The models included the digital controller in the loop. Computer assisted control system design techniques were used for the design of the controller parameters. The parameters were obtained by measuring the dynamic response of the model, including non-linearities. In order to be able to tune the PID controller parameters, estimation techniques were used. These were based on a performance index which measured the system error. The required criteria were to reach the desired position without overshooting and to minimise the effects of wind loading and variations in payloads. 


\section{CONCLUSIONS}

Simulation of the panel positioning robot has shown that it is feasible to develop such a device. In particular, the kinematic and dynamic simulation test-runs have shown that the configuration of the robot and its control strategies/servo-mechanisms (together with design modifications to the panel fixing mechanisms) allow the robot to work alongside a human operator in positioning and fixing panels onto a building's structure. The data generated by various simulation test-runs have helped to advance the design of this robotic device, including pinpointing necessary changes to its preliminary mechanical design. Kinematic and dynamic simulation bridges the gap between paper-based, desk studies and expensive prototyping.

\section{ACKNOWLEDGEMENT}

The authors grateful acknowledge the financial support of the Science and Engineering Research Council for the project, Opportunities for robotics in the construction of buildings for industry.

\section{REFERENCES}

1. Ibañez-Guzmán J., Atkin, B.L., Atkinson, P., Bayes, J. and Bridgewater, C.E., An Integrated Approach Towards Automating Construction, in Proc. 18th Intl. Assn. for Housing Science World Congress - Buildings in the Future, Rio de Janeiro, 1990.

2. Atkin, B.L., Atkinson, P., Bridgewater, C. E. and Ibañez-Guzmán J., A new direction in automating construction, in Proc. 6th Intl. Symp. on Robotics and Automation in Construction, Construction Industry Institute, San Francisco, 1989, pp119-186.

3. Bridgewater, C.E., Ibañez-Guzmán, J., Atkin, B.L. and Atkinson, P., Parts Set: Components of Modular Building Systems, in Proc. 7th Intl. Symp. on Robotics and Automation in Construction, Bristol Polytechnic, Bristol, 1990, pp 104-110.

4. Bridgewater, C.E., Atkin, B.L. Ibañez-Guzmán, J., and Atkinson, P., Design for automation: an operational framework, in Computer Integrated Construction, ed. $\mathrm{H}$ Wagter, Elsevier Science Publishers, in press, 8pp.

5. Ibañez-Guzmán J., Atkin, B.L., Atkinson, P., Bayes, J. and Bridgewater, C.E., Automation Systems and Robotic Tools for Modular Building Systems, Mechatronic Systems Engineering, 1, 1990, pp165-174.

6. Culviner, T., Graphics Windows for Concurrent Simulation, IEEE Computer Graphics and Applications, January 1989, pp8-9.

7. Donner, M., Computer simulation to aid robot selection, in Robot Technology, ed. A. Pugh, Peter Peregrinus Ltd., London, 1983.

8. Klein, C.A. and Maciejwski, A.A., Simulators, Graphic, in International Encyclopedia of Robotics: Applications and Automation, ed. R.C. Dorf, 3, John Wiley \& Sons, New York, 1988, pp1599-1608.

9. Fu, K.S., Gonzales, R.C. and Lee, C.S.G., Robotics: Control, Sensing, Vision and Intelligence, McGraw-Hill, New York, 1987.

10. GRASP User's Manual, BYG Systems, Nottingham, UK, 1988.

11. ROBCAD User's Manual, Tecnomatix Technologies, Novi, USA, 1989.

12. Luh, J.Y.S., An Anatomy of Industrial Robots and their Controls, IEEE Trans. in Automatic Control, February, 1983, pp133-153.

13. British Standards Institution, CP3 Chapter V: Part 2, Wind Loads, B.S.I., London.

14. Kabuka, M., and Escoto, R., Real Time Implementation of the Newton-Euler Equations of Motion on the NEC $\mu$ PD77230 DSP, IEEE Micro Magazine, 9, (1), February, 1989, pp66-76.

15. Snyder W.E., Industrial Robots: Computer Interfacing and Control, Prentice-Hall, Englewood Cliffs, N.J., 1985, pp89-96.

16. TUTSIM User's Manual Version 7.0, Meerman Automation, Netherlands, 1991. 